Bocker: Kohlenwasserstoffgehalt konz. Citronenole. 199

\title{
Die Bestimmung des Kohlenwasserstoffgehaltes konzentrierter Citronenöle;
}

\author{
von \\ E. Böoker.
}

[Mitteilung aus dem Laboratorium der Firma E. Sachsse \& Co.-Leipzig.]

Bekanntlich besteht das natürliche Citronenöl zum weitaus gröBten Teil aus geruchlich indifferenten Kohlenwasserstoffen, und zwar zu ca. $90 \%$ aus Terpenen und zu ca. $2 \%$ aus Sesquiterpenen. Von den restlichen $8 \%$ entfallen ca. $2 \%$ auf nichtflüchtige geruchlose Pflanzenwachsstoffe (Citrapten) und ca. $6 \%$ auf flüchtige sauerstoffhaltige Verbindungen, die eigentlichen Träger des Citronenaromas. Die Absonderung dieser Aromastoffe von der großen Masse indifferenter und in verdünntem Alkohol sehr schwer löslicher Begleitstoffe ist mehr und mehr zu einem praktischen Bedürfnis geworden, besonders im Interesse verschiedener $Z_{\text {weige }}$ der Getränkeindustrie, denen die Verwendung des in verdünntem Alkohol wenig löslichen natürlichen Citronenöls groBe Schwierigkeiten bereitete. So ist denn das "Konzentrieren" des natürlichen Citronenöls, d. h. die mehr oder minder vollständige Beseitigung der Kohlenwasserstoffe, ein wichtiger Fabrikationszweig für die Industrie der ätherischen Öle geworden. Im großen und ganzen kann man unter den Handelspräparaten drei Sorten unterscheiden: den höchsten Konzentrationsgrad weist das "terpen- und sesquiterpenfreie“ Citronenöl auf; es ist frei von allen Kohlenwasserstoffen und besteht ausschlieblich aus den Aromastoffen des natïrlichen Citronenöls. Den zweiten Grad vertritt das "terpenfreie" Citronenöl, das neben den Aromastoffen meist ca. 20-35\% Sesquiterpene enthält. An dritter Stelle endlich steht das sogenannte „konzentrierte" Citronenöl, das in der Regel zu etwa $20 \%$ aus Aromastoffen und zu etwa $80 \%$ aus Kohlenwasserstoffen, Terpenen und Sesquiterpenen, besteht.

Der wirtschaftliche Wert der durch Konzentrieren aus dem natürlichen Citronenöl erhältlichen Präparate richtet sich 
200 B $\delta$ cker: Kohlenwasserstoffgehalt konz. Citronenðle.

also nach dem Grade, bis zu welchem jeweils die Kohlenwasserstoffe entfernt worden sind. Es ist daher für die Wertbestimmung dieser Erzeugnisse von großem Nutzen, den Kohlenwasserstoffgehalt konzentrierter Citronenöle auf direktem Wege bestimmen zu können. Eine derartige Methode, an welcher es bislang fehlte, soll im folgenden beschrieben werden.

Das Wesen des Verfahrens besteht darin, da $B$ dem zu untersuchenden Öl die Aromastoffe entzogen werden. Das geschieht auf zweierlei Art: zuerst wird das Citral, das ca. ${ }^{2} / 3$ der gesamten Aromastoffe ausmacht, mittels Natriumsulfitlösung dem Gemisch entzogen. Das übrigbleibende Drittel Aromastoffe wird durch Auswaschen mit verdünntem Alkohol entfernt, dessen Stärke so gewählt ist, dab die Kohlenwasserstoffe bei Einhaltung gewisser Kautelen fast gänzlich ungelöst hinterbleiben. - Der Gung des Verfabrens ist folgender:

$10 \mathrm{ccm}$ des zu untersuchenden Öls werden mittels Pipette in einen Cassiakolben gebracht, in welchem dann nach der Methode von Burgess mittels 40prozent. frischer Natriumsulfitlösung die Citralbestimmung ausgeführt wird. Sollte wie in der Regel zu erwarten - das von der Sulfitlösung nicht aufgenommene Öl weniger als $6 \mathrm{ccm}$ betragen, so wird noch eine zweite Citralbestimmung mit 5 oder, wenn erforderlich, $10 \mathrm{ccm}$ Öl ausgeführt. Das unangegriffene Öl beider Citralbestimmungen wird vereinigt. $5 \mathrm{ccm}$ dieses citralfreien Öls werden nun mittels Pipette in einen Scheidetrichter von etwa $600-700 \mathrm{ccm}$ Inhalt gebracht, in den unmittelbar vorher $500 \mathrm{ccm}$ auf $0^{\circ}$ bis $-2^{\circ}$ abgekühlter Sprit von genau 51 Volumprozenten (Tralles) eingefült waren. Der Scheidetrichter wird sofort mit straffem Korkstopfen verschlossen und mehrmals umgeschüttelt, so daB das Öl in sehr feine Verteilung gebracht wird. Dabei gehen die Aromastoffe des Citronenöls in Lösung, während die Kohlenwasserstoffe beinahe völlig unangegriffen bleiben. Nach dem Schütteln wird der Scheidetrichter (Kork nach unten) in ein Kaltebad von kleinen Eis-

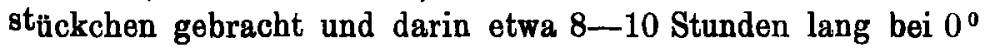
belassen. Während dieser Zeit sammeln sich die staubfeinen Oltröpfchen an der Oberfläche der Spritlösung und schließen sich dort zu gröBeren Tropfen zusammen. Der Scheidetrichter wird nach der angegebenen Zeit aus dem Kältebad heraus- 
Bðcker: Kohlenwasserstoffgehalt konz. Citronenðle. 201

genommen, langsam in die richtige Lage aufgerichtet und in ein Stativ gehängt. Man wartet nun ab, bis die Spritlösung sich so weit geklärt hat, daB höchstens noch ein leichter Schleier bemerkbar ist ${ }^{1}$ ), und läBt dann den Sprit langsam bis auf etwa $10 \mathrm{ccm}$ ab. Nun spült man die an der Trichterwand etwa haften gebliebenen Öltröpfehen mit eiskaltem 50 prozent. Sprit nach, wobei man sich zweckmäBig einer 10 ccm-Pipette bedient, deren Auslaufspitze man an der innern Trichterhalswandung im Kreis herumfüht. Auf diese Weise hat man alles ungelöst gebliebene Öl im untersten spitz zulaufenden Teil des Scheidetrichters versammelt. Man wartet wieder völlige Klärung des durch die Nachspülung trübe gewordenen Sprits ab und läBt dann den Sprit so weit als möglich ab. Das im Scheidetrichter verbleibende, zum Teil noch schaumige Öl überläbt man zunächst der Klärung, um es dann vorsichtig in einen mit Zehntelkubikzentimetereinteilung versehenen Mebzylinder abzulassen. Dabei wird wieder mit etwas eiskaltem 51 prozent. Sprit nachgespült. Das Ausflubrchr des Scheidetrichters muB kurz und eng sein, um den quantitativen Ubbergang des Öls in den ebenfalls eng zu wählenden Mebzylinder (Rohrweite etwa wie beim Cassiakolben, oberer Teil trichterförmig erweitert) zu erleichtern. Sehr zweckmäBig ist es, für diese Bestimmung sich einen Scheidetrichter anfertigen zu lassen, dessen unterer Teil in ein graduiertes Rohr übergeht. Dadurch erübrigt sich das Utberführen des ungelösten Ols in einen besonderen MeBzylinder.

Nachdem sich das Öl vollkommen geklärt hat - bei hartnäckigem Schaum gebe man einige Tropfen verdünnte Essigsäure hinzu -, wird sein Volumen abgelesen. Die Berechnung des Resultats geschieht in der Weise, daB man sich die angewandten $5 \mathrm{ccm}$ citralfreien Öls durch das dazugehörige Quantum Citral zum ursprünglichen Öl ergänzt denkt und nun auf dies vergröBerte Volum das ermittelte Volum ungelösten Öls bezieht. Hätte z. B. die Citralbestimmung 50\% Citral ergeben und wären von den angewandten $5 \mathrm{ccm}$ citralfreien Öls $2,5 \mathrm{ccm}$ ungelöst zurückgeblieben, so wären diese $2,5 \mathrm{ccm} \mathrm{zu}$

1) Die Klarung erfordert meist $1 / 2$ Tag, es kann aber auch 1 bis 2 Tage dauern, zumal, wenn das untersuchte Öl bereits etwas verharzt ist. 
202 Bocker: Kohlenwasserstoffgehalt konz. Citronenøle.

beziehen auf ein Gesamtvolum von $10 \mathrm{ccm}$ ursprünglichen Öls, der ermittelte Kohlenwasserstoffgehalt betrüge also 25 Volumprozent.

Das vorstehend beschriebene Verfahren ist an einer ganzen Reibe von Versuchsmischungen mit gutem Erfolg erprobt worden. Zur Herstellung dieser Mischungen dienten Citronenterpene, Citronensesquiterpene und völlig terpen- und sesquiterpenfreies Citronenöl. Nachstehend die Eigenschaften dieser drei Komponenten.

Citronenterpene:

\begin{tabular}{|c|c|}
\hline 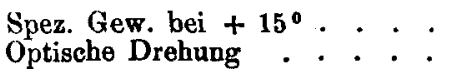 & $\begin{array}{l}\cdot=0,8515 \\
\cdot=+63^{\circ} 30^{\prime}\end{array}$ \\
\hline \multicolumn{2}{|l|}{ Citronensesquiterpene: } \\
\hline 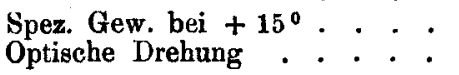 & $\begin{array}{l}=0,8980 \\
\cdot=-33^{\circ}\end{array}$ \\
\hline \multicolumn{2}{|c|}{ Citronenöl terpen- und sesquiterpenfrei : } \\
\hline $\begin{array}{l}\text { Spez. Gew. bei }+15^{\circ} \cdot . \cdot . \\
\text { Optische Drehung. } . \text {. } \\
\text { Löslich in 69 volumprozent. Sprit } \\
\text { Löslich in 60volumprozent. Sprit } \\
\text { Citralgehalt (nach Burgess) }\end{array}$ & 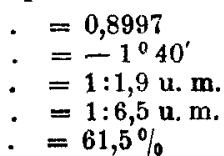 \\
\hline
\end{tabular}

Um nicht bei jeder einzelnen Versuchsmischung die Citralbestimmung ausführen zu müssen, habe ich gleich $200 \mathrm{ccm}$ des terpen- und sesquiterpenfreien Citronenöls nach der Burgessschen Methode rom Citral befreit und das hierbei erhaltene citralfreie Öl für die einzelnen Versuchsmischungen verwandt, an denen dann natürlich nur noch die Spritwaschmethode auszuführen war. Die Entcitralisierung geschah in zwei Portionen zu $100 \mathrm{ccm}$ im 3-Literrundkolben im Wasserbad, dazu wurden je $400 \mathrm{~g}$ Natriumsulfit, frisch gelöst in $600 \mathrm{~g}$ Wasser, verwandt. Die Neutralisierung der frei werdenden Natronlauge geschah mittels 20prozent. Essigsäure. Der ProzeB war nach etwa 1 Stunde beendet. Die $200 \mathrm{ccm}$ terpen- und sesquiterpenfreies Citronenöl ergaben $80 \mathrm{ccm}$ citralfreies Öl, was einem Citralgehalt von 60 Volumprozent entspricht. Das citralfreie Öl besaB folgende Eigenschaften:

$$
\begin{aligned}
& \text { Spez. Gew. bei }+15^{\circ} \text {. . . . . }=0,9117 \\
& \text { Optische Drehung. . . . . . . }=-3^{\circ} 13^{\prime} \\
& \text { Löslich in } 69 \text { volumprozent. Sprit . }=1: 2 \mathrm{u} . \mathrm{m} \text {. } \\
& \text { Löslich in } 60 \text { volumprozent. Sprit . }=1: 15 \mathrm{u} \text {. m. }
\end{aligned}
$$

Die nachfolgende Zusammenstellung zeigt, welche Resultate die neue Methode bei den Versuchsmischungen lieferte. Bei 
Bocker: Kohlenwasserstoffgehalt konz. Citronenole. 203

allen Mischungen ist der Einfachheit halber nur die Kohlenwasserstoffkomponente aufgeführt, den Rest bis $100 \%$ nimmt stets das citralbefreite terpen- und sesquiterpenfreie Citronenöl ein. Die in [] gesetzten Prozentzahlen bedeuten den auf ursprüngliches citralhaltiges Öl berechneten Kohlenwasserstoffgehalt der Mischungen.

\begin{tabular}{|c|c|c|c|c|}
\hline \multicolumn{3}{|c|}{ Angewandt } & \multicolumn{2}{|c|}{ Ermittelt } \\
\hline $\begin{array}{r}\text { Misch. 1: } 80 \% \\
\Rightarrow \quad 2: 80 \%\end{array}$ & $\left\{\begin{array}{l}61,5] \\
{[61,5]}\end{array}\right\}$ & $\begin{array}{l}\text { Citronen- } \\
\text { terpene }\end{array}$ & $\begin{array}{lll}74 & \% & {[56,92]} \\
72 & \% & {[55,38]}\end{array}$ & $\begin{array}{l}\text { Kohlenwasser- } \\
\text { stoffe }\end{array}$ \\
\hline $\begin{array}{l}\Rightarrow \quad 3: 80 \% \\
\Rightarrow \quad 4: 80 \%\end{array}$ & $\{61,5]\}$ & $\begin{array}{c}\text { Citronen } \\
\text { sesquiterpene }\end{array}$ & 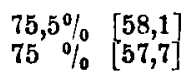 & $\begin{array}{l}\text { Kohlenwasser- } \\
\text { stoffe }\end{array}$ \\
\hline $\begin{array}{l}\Rightarrow \quad 5: 40 \% \\
" \quad 6: 40 \%\end{array}$ & $[21,05]\}$ & $\begin{array}{c}\text { Citronen- } \\
\text { terpene }\end{array}$ & $\begin{array}{l}37,5 \%[19,73] \\
36,5 \%\end{array}$ & $\begin{array}{l}\text { Kohlenwasser- } \\
\text { stoffe }\end{array}$ \\
\hline $\begin{array}{l}7: 40 \% \\
" \quad 8: 40 \%\end{array}$ & $\begin{array}{l}{[21,05} \\
{[21,05]}\end{array}$ & $\begin{array}{c}\text { Citronen- } \\
\text { sesquiterpene }\end{array}$ & $\begin{array}{l}42,5 \%[22,36] \\
39 \%[20,52]\end{array}$ & $\begin{array}{l}\text { Kohlenwasser- } \\
\text { stoffe }\end{array}$ \\
\hline $\begin{array}{r}9: 20 \% \\
" 10: 20 \%\end{array}$ & $\begin{array}{l}{[9,09]} \\
{[9,09]}\end{array}$ & $\begin{array}{l}\text { Citronen- } \\
\text { terpene }\end{array}$ & $\begin{array}{ll}20,5 \% & {[9,31]} \\
18 \% & {[8,18]}\end{array}$ & $\begin{array}{c}\text { Kohlenwasser- } \\
\text { stoffe }\end{array}$ \\
\hline $\begin{array}{l}\text { "11: } 20 \% \\
" 12: 20 \%\end{array}$ & $\left.\begin{array}{l}{[9,09]} \\
{[9,09]}\end{array}\right\}$ & $\begin{array}{c}\text { Citronen- } \\
\text { sesquiterpene }\end{array}$ & $\left.\begin{array}{ccc}22 & \% & {[10]} \\
20 & \% & {[9,09}\end{array}\right]$ & $\begin{array}{l}\text { Kohlenwasser- } \\
\text { stoffe }\end{array}$ \\
\hline $\begin{array}{l}\text {, 13: } 10 \% \\
\Rightarrow 14: 10 \%\end{array}$ & $\left.\begin{array}{l}{[4,25]} \\
{[4,25]}\end{array}\right\}$ & $\begin{array}{l}\text { Citronen- } \\
\text { terpene }\end{array}$ & $\begin{array}{lrr}9 \% & {[3,83]} \\
7,5 \% & {[3,19]}\end{array}$ & $\begin{array}{c}\text { Kohlenwasser- } \\
\text { stoffe }\end{array}$ \\
\hline $\begin{array}{l}\text { "15: } 10 \% \\
" 16: 10 \%\end{array}$ & {$\left[\begin{array}{l}4,25] \\
4,25]\end{array}\right.$} & $\begin{array}{c}\text { Citronen- } \\
\text { sesquiterpene }\end{array}$ & $\begin{array}{ll}10,5 \% & {[4,46]} \\
10,5 \% & {[4,46]}\end{array}$ & $\begin{array}{c}\text { Kohlenwasser- } \\
\text { stoffe }\end{array}$ \\
\hline $\begin{array}{l}\text { "17: } 5 \% \\
" 18: 5 \%\end{array}$ & $\left.\begin{array}{l}2,06] \\
2,06]\end{array}\right\}$ & $\begin{array}{l}\text { Citronen- } \\
\text { terpene }\end{array}$ & $\left.\begin{array}{ll}3,5 \% & {[1,44} \\
3,5 \% & {[1,44}\end{array}\right]$ & $\begin{array}{l}\text { Kohlenwasser- } \\
\text { stoffe }\end{array}$ \\
\hline $\begin{array}{l}" 19: 5 \% \\
" 20: 5 \%\end{array}$ & $\begin{array}{l}{[2,06]} \\
{[2,06]}\end{array}$ & $\begin{array}{c}\text { Citronen- } \\
\text { sesquiterpene }\end{array}$ & $\begin{array}{ccc}4,5 \% & {[1,85]} \\
4 \% & {[1,73]}\end{array}$ & $\begin{array}{c}\text { Koblenwasser- } \\
\text { stoffe }\end{array}$ \\
\hline
\end{tabular}

Die erhaltenen Werte sind bei den Versuchsmischungen, bei denen Citronenterpene verwandt sind, fast durchweg etwas zu niedrig. Das hängt damit zusammen, daB die Terpene in verdunnten Spriten etwas besser löslich sind als die Sesquiterpene. Immerhin scheint mir aus den vorstehenden Versuchen hervorzugehen, daB die Methode für die quantitative Bestimmung des Kohlenwasserstoffgehalts konzentrierter Citronenöle brauchbar ist. Ich habe diese Methode denn auch an zahlreichen Handelsölen zur Anwendung gebracht. Nachstehend einige Beispiele:

Ein terpenfreies Citronenöl eigner Fabrikation ergab folgende Werte: 
204 Bocker: Kohlenwasserstoffgehalt konz. Citronenole.

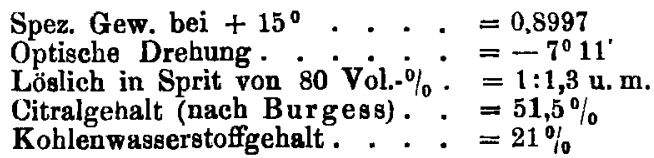

Ein terpen- und sesquiterpenfreies Citronenöl eigner Fabrikation lieferte folgende Daten:

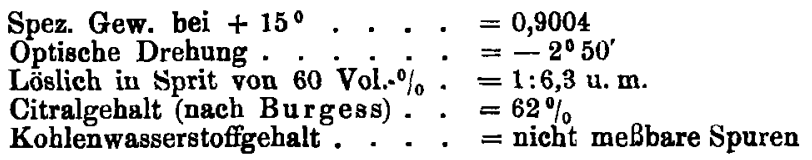

Ein als terpenfrei bezeichnetes Citronenöl vom englischen Markte zeigte folgendes Bild:

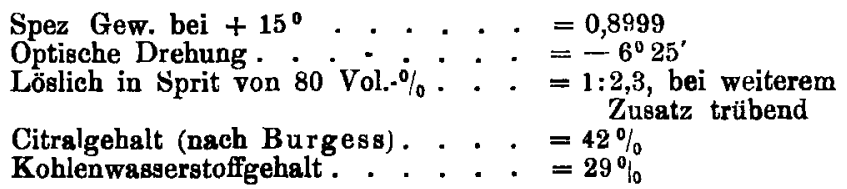

Ein aus Italien stammendes, ebenfalls als terpenfrei bezeichnetes Citronenöl besa $B$ folgende Eigenschaften:

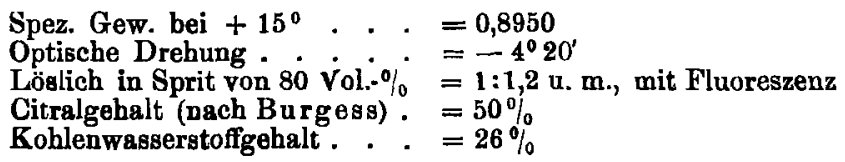

Mit ihrem Kohlenwasserstoffgehalt von 25-30\% stellen die letztgenannten beiden Ole den Durchschnittstyp der terpenfreien Citronenöle des Handels auf, wie sie normalerweise durch Entfernung aller Terpene aus dem natürlichen Citronenöle erhalten werden. Berücksichtigt man, $\mathrm{da} B$ das natürliche Citronenöl (wie eingangs erwähnt) ca. $6 \%$ Aromastoffe und ca. $2 \%$ Sesquiterpene enthält, so kann man nicht umbin, einen Kohlenwasserstoffgehalt (in diesem Fall Sesquiterpengehalt) von 25-30\% beim terpenfreien Citronenöl als erlaubt zu bezeichnen. Wesentlich anders sind indes die Erfahrungen, die ich an Handelsölen machte, welche ihrer Bezeichnung nach keine Kohlenwasserstoffe mehr enthalten sollten. Wie aus den nachfolgenden Analysen hervorgeht, wiesen diese Öle entgegen ihrer ausdrücklichen Bezeichnung ,sesquiterpenfrei" zum Teil sehr beträchtliche Mengen von Sesquiterpenen anf.

Ein Öl deutschen Ursprungs besaB folgende Eigenschaften:

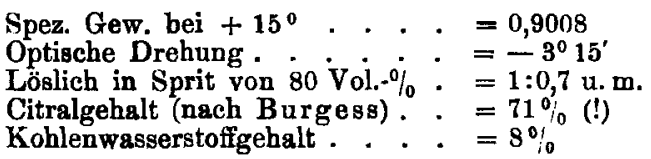


Brcker: Koblenwasserstoffgehalt konz. Citronenöle. 205

Ein italienisches Öl lieferte folgende Werte:

$$
\begin{aligned}
& \text { Spez. Gew. bei }+15^{\circ} \text {. . . . }=0,8968 \\
& \text { Optische Drehung . . . . . }=-5^{\circ} 50^{\circ} \\
& \text { Löslich in Sprit von } 69 \text { Vol.. } \%=1: 27 \mathrm{u} . \mathrm{m} \text {. } \\
& \text { Citralgehalt (nach Burgess). } .=62 \% \\
& \text { Kohlenwasserstoffgehalt . . . . }=12 \%
\end{aligned}
$$

Ein Öl vom englischen Markt gab folgendes Bild:

$$
\begin{aligned}
& \text { Spez. Gew. bei }+15^{\circ} \text {. . . . }=0,8983 \\
& \text { Optische Drehung . . . . . . }=-3^{0} 10^{\prime} \\
& \text { Löslich in Sprit von } 80 \mathrm{Vol} .-\%=1: 1 \mathrm{u} . \mathrm{m} \text {. } \\
& \text { Nicht klar löslich in Sprit von } 69 \text { Vol. } \% \\
& \text { Citralgehalt (nach Burgess). . }=52 \% \\
& \text { Kohlenwasserstoffgehalt . . . : }=22 \% \text { (!) }
\end{aligned}
$$

Besonders dieser letzte Fall beweist schlagend die Notwendigkeit einer Methode zur quantitativen Bestimmung des Kohlenwasserstoffgebalts konzentrierter Citronenöle. Der Umstand, daB zu dieser Untersuchung nur geringe Mengen (10 bis $20 \mathrm{ccm}$ ) des teuren Materials erforderlich sind, ermöglicht die Anwendung dieser Methode wohl in den allermeisten Fällen. Sollten größere Materialmengen zur Verfügung stehen, so kann man das oben beschriebene Verfahren nach der Richtung vervollständigen, daB man auBer der Menge auch Art und Eigenschaften der abgesonderten Kohlenwasserstoffe analytisch ermittelt. Hierzu verfährt man zweckmäBig folgendermaßen:

$100-200 \mathrm{ccm}$ des zu untersuchenden Öls werden einer fraktionierten Destillation im luftverdünnten Raum unterworfen. Dabei gehen etwa vorhandene Terpene in den ersten Fraktionen uber. Man destilliert nur so lange, als die Fraktionen (die man etwa $10 \mathrm{ccm}$ groB wählt) noch Rechtsdrehung aufweisen. Diese rechtsdrehenden Fraktionen vereinigt man als „terpenhaltigen Anteil", der ganze Rest des Ols bildet den "sesquiterpenhaltigen Anteil". Jeder dieser beiden Anteile wird nun gesondert nach der Burgessschen Methode rom Citralgehalt befreit. Die übrigbleibenden Ölmengen werden dann, ebenfalls gesondert, mit der hundertfachen Menge eiskalten 51 prozent. Sprits in der gleichen Weise behandelt, wie oben ausgeführt. An Stelle des Scheidetrichters treten natürlich gröBere Glas. flaschen, die man nach beendetem Schütteln in Kältebäder stellt, bis sich die etwa ungelöst bleibenden Ölteilchen an der Oberfläche gesammelt haben. Nach völliger Klärung..des Sprits wird vorsichtig abgehebert. Die abgeschiedenen Öle werden gesondert gesammelt und ihrem Volum nach bestimmt. Aus den so erhaltenen Mengen berechnet man dann in der oben beschriebenen Weise einerseits den Gehait an Terpenen (erhalten aus dem "terpenhaltigen Anteil"), andererseits den Gehalt an Sesquiterpenen (erhalten aus dem "sesquiterpenhaltigen Anteil"). Um sie noch näher als solche zu identi- 
fizieren, untersucht man sie auf ihr optisches Drehungsvermögen. Die Citronenterpene (Hauptbestandteil d-Limonen) weisen starke Rechtsdrehung, die Citronensesquiterpene (Hauptbestandteil das linksdrehende Bisabolen) ${ }^{1}$ ) beträchtliche Linksdrehung auf. Weitere Identifizierung kann einerseits durch Darstellung des bekannten Limonentetrabromids, andererseits durch Überführung in das Bisabolentrichlorhydrat ${ }^{1}$ ) erfolgen.

Ich habe nach dieser Methode eine Versuchsmischung $(200 \mathrm{ccm})$ untersucht, die zu 5 Vol.-Proz. aus Citronenterpenen, zu $20 \%$ aus Citronensesquiterpenen und zu $75 \%$ aus terpenund sesquiterpenfreiem Citronenöl bestand. Die Mischung besa $B$ folgende Eigenschaften:

$$
\begin{aligned}
& \text { Spez. Gew. bei }+15^{\circ} \text {. . . . . }=0,8971 \\
& \text { Optische Drehung . . . . . } .^{\circ}=-4^{0} 25 \\
& \text { Löslich in } 80 \text { volumprozent. Sprit . }=1: 0,9 \text { u. m. }
\end{aligned}
$$

Bei der fraktionierten Destillation erhielt ich 4 Fraktionen zu je $10 \mathrm{ccm}$ mit optischer Rechtsdrehung. Diese $40 \mathrm{ccm}$ wurden als "terpenhaltiger Anteil" vereinigt, optische Drehung $=+13^{\circ} 40^{\prime}$. Der Rest, ca. $160 \mathrm{ccm}$,sesquiterpenhaltiger Anteil", wies eine Linksdrehung von $6^{\circ} 15^{\prime}$ auf. Durch Behandlung mit frischer 40 prozent. Natriumsulfitlösung nach der Methode von Burgess wurden insgesamt $94 \mathrm{ccm}$ Citral $=47$ Vol. Proz. aus beiden Anteilen entfernt, nämlich $14 \mathrm{ccm}$ aus dem terpenhaltigen, $80 \mathrm{ccm}$ aus dem sesquiterpenhaltigen Anteil. Die Extraktion mit eiskaltem 51 prozent. Sprit lieferte aus ersterem Anteil $7,8 \mathrm{ccm}$ Terpene $=3,9 \%$, aus letzterem Anteil 37,6 ccm Sesquiterpene $=18,8 \%$. Es waren also insgesamt 22,7 Vol.-Proz. Kohlenwasserstoffe ermittelt, während 25 Vol.-Proz. im Versuchsöl wirklich enthalten waren. Sicherlich ein recht befriedigendes Ergebnis.

Das optische Drehungsvermögen der zurückerhaltenen Terpene war freilich nicht unbeträchtlich geringer, als das der zur Versuchsmischung verwandten, nämlich $+51^{\circ}$ gegenüber $+63^{\circ} 30^{\circ}$. Ebenso zeigten auch die wiedererhaltenen Sesquiterpene nicht mehr ihre ursprüngliche Linksdrehung von $\mathbf{3 3}^{\mathbf{0}}$, sondern eine solche von nur $26^{\circ} 35^{\prime}$. Es hängt dies damit zusammen, daB die abgeschiedenen Kohlenwasserstoffe etwas Sprit und auch Spuren von sauerstoffhaltigen Verbindungen des Citronenöls enthalten, während andererseits der Sprit auch geringe Mengen von Kohlenwasserstoffen in sich aufnimmt. Es findet also bis zu einem gewissen Grade ein Austausch von Kohlenwasserstoffen gegen Sprit und Spuren sauerstoffhaltiger Verbindungen statt. Durch Verwendung des Sprits in eiskaltem Zustande ist dieser Austausch zwar auf ein Mini-

1) Berichte von Schimmel \& Co., Oktober 1909, S. 50. 
mum reduziert, ganz ausschalten läßt er sich jedoch nicht. Um die abgeschiedenen Kohlenwasserstoffe in noch reinerer Form zu erhalten, wäscht man sie mit etwa der 20 fachen Menge eiskalten 51 prozent. Sprits und dann mit etwa der 5 fachen Menge destillierten Wassers nach. Auf diese Weise erhielt ich 6,2 ccm Terpene von $+57^{\circ}$ und $34,3 \mathrm{ccm}$ Sesquiterpene von $-27^{\circ} 45^{\prime}$ optischer Drehung.

Auch an einem Handelspräparat habe ich diese modifizierte Bestimmungsmethode mit bestern Erfolg in Anwendung gebracht, und zwar an dem .als "sesquiterpenfrei" bezeichneten aus England bemusterten Öl, dessen ich bereits oben Erwähnung getan. In diesem Öl, von dem mir $100 \mathrm{~g}$ zur Verfügung standen, konnte ich $3,2^{\circ} \%$ Terpene $\left(\alpha_{D}=+52^{\circ}\right)$ und $17,4^{\%} \%$ Sesquiterpene $\left(\alpha_{D}=-28^{\circ} 15^{\prime}\right)$, also insgesamt 20,6 Vol.Proz. Kohlenwasserstoffe nachweisen. Dies Resultat stimmt also recht gut mit dem an $15 \mathrm{ccm}$ des gleichen Oles nach der einfachen Methode erhaltenen Befund von $22 \%$ Kohlenwasserstoffen (s. oben) überein. $1 \mathrm{~g}$ der Sesquiterpene benutzte ich dazu, das Bisabolentrichlorhydrat daraus herzustellen. Ich löste das Gramm, das sich in einem $15 \mathrm{ccm}$-Erlenmeyerkölbchen befand, mit $5 \mathrm{ccm}$ wasserfreien Schwefeläthers und leitete in die in Eiswasser gestellte Lösung trockenes Chlorwasserstoffgas bis zur Sättigung ein. Die Lösung nahm dabei ein dunkelbraune Färbung an. Dann vertrieb ich den Schwefeläther auf dem Wasserbade und fügte ca. $5 \mathrm{ccm} 90$ prozent. Sprit zum heiBen Rückstande. Beim Erkalten schieden sich sehr reichliche Mengen des Bisabolentrichlorhydrats aus, das nach zweimaligem Umkrystallisieren aus 90 prozent. Alkohol den korrekten Schmelzpunkt $+79,4^{\circ}$ zeigte. Als Nebenprodukt wurden geringe Mengen eines öligen, in 90 prozent. Alkohol schwer löslichen Körpers erhalten, in welchem man wohl ein unreines Chlorhydrat des begleitenden Sesquiterpens Cadinen ${ }^{1}$ ) vermuten darf. - Von diesem reichlich 20 Vol.-Proz. wertlose Kohlenwasserstoffe enthaltenden, ganz zu Unrecht als ,sesquiterpenfrei" bezeichneten Citronenöl hatte eine englische Autorität in einem vielverbreiteten Gutachten behauptet, es sei „frei von Terpenen und Sesquiterpenen"!

Es ist wahrscheinlich, daB in ähnlicher Weise, wie bei dem hier beschriebenen Verfahren, sich auch bei anderen konzentrierten ätherischen Ölen der Gebalt an Kohlenwasserstoffen wird bestimmen lassen. Dahin zielende Versuche habe ich bereits in Angriff genommen.

1) Berichte von Schimmel \& Co., Okt. 1909, S. 51. 